\section{Acessibilidade organizacional de crianças com paralisia cerebral à reabilitação motora na cidade do Recife}

\section{Organizational access to motor rehabilitation for children with cerebral paralysis in the city of Recife}

Aleide Karine Vieira Tôrres 1

Silvia Wanick Sarinho 2

Katia Virginia de Oliveira Feliciano 3

Maria Helena Kovacs 4

1 Programa de Pós-graduação em Saúde da Criança e do Adolescente. Universidade Federal de Pernambuco. Recife, PE, Brasil.

2,4 Faculdade de Ciências Médicas. Universidade de Pernambuco. Av. Agamenon Magalhães, s. n. Santo Amaro. Recife, PE, Brasil.

CEP: 50.100-010. E-mail: silviaws@gmail.com

3 Instituto de Medicina Integral Prof. Fernando Figueira. Recife, PE, Brasil.

\begin{abstract}
Objectives: to characterize organizational access to motor rehabilitation services for children with cerebral paralysis.

Methods: a descriptive, retrospective, censusbased study, carried out between January and June 2009, at three rehabilitation services in Recife. The study covered 38 children aged less than five years, with the disorder, residing in Recife. A questionnaire was used to obtain information from the individual accompanying the child and the child's medical records. The variables were organized according to current service, first service used, and number of services used.

Results: of the people accompanying the children, $94.7 \%$ were one of the child's parents or legal guardian (76.3\% mother) and $68.4 \%$ of the children were aged between 25 and 59 months. Half had attended more than one service, in $28.2 \%$ of cases simultaneously. For $28.2 \%$ rehabilitation was commenced more than six months after diagnosis. The children were mostly referred to the service by a doctor $(75.8 \%)$ and $86.4 \%$ had a maximum waiting time of 30 days for the first consultation with a physiotherapist. The parents or legal guardians generally found it difficult to schedule the first consult (68.4\%). Greater difficulty in continuing physiotherapy was found among those who used more than one service.

Conclusions: there appears to be a hidden demand. Use of more than one service (at times simultaneously) suggests dissatisfaction with the level of care. Political and organizational processes relating to the referral/counter-referral system should be accorded high priority.
\end{abstract}

Key words Cerebral palsy, Rehabilitation, Health services accessibility, Child health (Public health)

\section{Resumo}

Objetivos: caracterizar acessibilidade organizacional de crianças com paralisia cerebral aos serviços de reabilitação motora.

Métodos: estudo descritivo, retrospectivo, censitário, efetuado de janeiro a junho/2009, em três serviços de reabilitação do Recife. Participaram 38 menores de cinco anos, portadores do agravo, residentes no Recife. Utilizou-se questionário para obter informações de acompanhantes e prontuários das crianças. As variáveis foram descritas segundo serviço atual, primeiro serviço utilizado e número de serviços utilizados.

Resultados: entre os acompanhantes, 94,7\% eram responsáveis pela criança $(76,3 \%$ mãe) e $68,4 \%$ das crianças tinham entre 25-59 meses de idade. Metade usou mais de um serviço, ocorrendo utilização simultânea em 26,3\%. Para 28,2\% reabilitação iniciou-se mais de seis meses após diagnóstico. As crianças foram encaminhadas por médico $(75,8 \%) e$ 86,4\% tiveram um tempo máximo de espera para primeira consulta com fisioterapeuta de 30 dias. As responsáveis encontraram dificuldade para agendar primeira consulta $(68,4 \%)$. Uma maior dificuldade para continuar fisioterapia foi identificada entre aqueles que usaram de dois e mais serviços.

Conclusões: constatou-se demanda reprimida. Utilização de mais de um serviço e superposição de serviços sugeriram insatisfação com atenção. Os processos políticos e organizacionais voltados à estruturação do sistema de referência/contrarreferência necessitam priorização.

Palavras-chave Paralisia cerebral, Reabilitação, Acessibilidade aos serviços de saúde, Saúde da criança 


\section{Introdução}

A Organização das Nações Unidas afirma que, no Mundo, aproximadamente 600 milhões de pessoas apresentam deficiência, das quais $80 \%$ vivem nos países em desenvolvimento. 1 Conforme Censo Demográfico de 2000, no Brasil, 24,5 milhões de pessoas manifestam algum tipo de deficiência ${ }^{2}-$ $48 \%$ portam deficiência física e $22,9 \%$ motora constituindo grupo heterogêneo que inclui as crianças com paralisia cerebral (PC). Doença que mostra tendência mundial ao crescimento, sobretudo, pela maior sobrevivência de prematuros.3,4 Inexistem estudos no país sobre a frequência deste agravo, estimando-se que ocorram entre 30.000 e 40.000 novos casos por ano. 5

A PC é uma condição crônica que exige tratamento de equipe multidisciplinar ao longo da vida.6,7 Principal enfoque terapêutico, a fisioterapia motora precisa ser precoce e continuada, requerendo serviços especializados que garantam assistência qualificada. ${ }^{8}$ A Portaria $n^{\circ} 827 / 1991$, o Decreto $n^{\circ}$ 3.298/1999 e a Portaria $n^{\circ} 1060 / 2002$ do Ministério da Saúde estabelecem como competência do Sistema Único de Saúde (SUS) viabilizar a integralidade da atenção aos portadores de deficiência, propiciando acesso às ações de saúde específicas da condição, inclusive concessão de órteses e próteses, além das ações voltadas às doenças e agravos comuns à infância. 9

O conceito de acesso é complexo com variações entre autores e mudanças ao longo do tempo de acordo com o contexto, bem como apresenta variações na terminologia empregada. Alguns autores recorrem ao termo acessibilidade que traduz o ser acessível, já outros preferem o substantivo acesso como indicativo do ato de ingressar, dar entrada. O acesso também é considerado como o uso de serviço em tempo adequado para alcançar o melhor resultado possível, não distinguindo entre acesso e uso. Ainda pode significar o ato de ingressar no serviço e dar continuidade ao tratamento. 10

Para Donabedian, ${ }^{11}$ a acessibilidade denota o grau de facilidade para obtenção dos cuidados de saúde necessários, contemplando duas dimensões inter-relacionadas e complementares: a sócio-organizacional ou funcional (a disponibilidade de serviços, a oferta de atividades, os horários de atendimento, os modos de agendamento das consultas, os critérios formais ou informais adotados para selecionar a demanda, o sistema de referência e contrarreferência, o grau de ajuste entre as necessidades dos pacientes e os recursos utilizados na assistência à saúde) e a geográfica (o tempo gasto no desloca- mento da residência do usuário ao serviço de saúde e o custo da viagem). Além destas, convém considerar a acessibilidade sociocultural (os aspectos que influenciam a percepção das necessidades de saúde e a busca de cuidados). ${ }^{10,12-14}$

Estudos constataram, no Canadá, longos períodos de espera para reabilitação motora de crianças com deficiência. ${ }^{15-18}$ Em Ontário, 23\% dos menores de cinco anos com retardo no desenvolvimento não efetuavam fisioterapia motora seis meses após encaminhamento. 16 Nos quatro principais centros de referência de Montreal, crianças com deficiência física esperavam, em média, quatro meses para início da fisioterapia motora e, quando encaminhadas pelas duas principais unidades de referência pediátrica, demoravam ao redor de sete meses para iniciar a fisioterapia. 18

É inquestionável a importância de identificar barreiras no ato de ingressar aos serviços, dados os custos sociais e humanos advindos do não atendimento em tempo hábil. Pesquisa efetuada por Meio et al., 19 na cidade do Rio de Janeiro, para avaliar encaminhamento de crianças com deficiência à reabilitação motora, evidenciou que a demanda é maior do que a oferta de serviços especializados para prestação qualificada desse tipo de atendimento, gerando demora no início do tratamento. Resultado que, segundo os autores, sugeria estrutura desarticulada e insuficiente da rede de assistência reabilitadora.

Para Bradley et al.,20 o difícil acesso das pessoas com deficiência aos serviços necessários tende a potencializar a deterioração provocada pelo agravo, levando ao aparecimento de condições secundárias que contribuem para a diminuição da qualidade de vida, no tocante às dimensões físicas, psicológicas e sociais do bem-estar delas. 8,21,22 Também pode, em longo prazo, aumentar os custos da atenção à saúde e do suporte essencial dos serviços sociais. ${ }^{23}$ Neste contexto, tomando como referência o conceito de Donabedian, ${ }^{11}$ este estudo buscou caracterizar a acessibilidade organizacional de crianças portadoras de PC aos serviços de reabilitação motora, da rede complementar do SUS, na cidade do Recife.

\section{Métodos}

Estudo descritivo, retrospectivo, censitário, realizado em três serviços da rede complementar credenciados para assistência físioterapêutica motora aos usuários do SUS, no Recife: Grupo Universitário de Reabilitação Infantil (GURI) e Centro de Reabilitação e Valorização da Criança (CERVAC), com oferta de atendimento todos os dias da semana 
nos dois turnos de trabalho, e Serviço Especializado de Reabilitação (SER), cuja disponibilidade está restrita ao turno da tarde.

Após cuidadosa avaliação das informações contidas nos prontuários, em cada um dos serviços preparou-se listagem de menores de cinco anos de idade, com PC, residentes no Recife, que realizavam reabilitação motora durante coleta de dados, entre janeiro e junho de 2009, independentemente do tipo de PC, presença de disfunções associadas e doenças secundárias. Das 44 crianças que atenderam aos critérios de inclusão, 38 participaram do estudo. As seis perdas decorreram de desligamento dos serviços por faltas frequentes e solicitação materna.

A coleta de dados foi realizada por uma das autoras (A.K.V.T.), utilizando instrumento com respostas provenientes de duas fontes de informação: a) as pessoas que traziam a criança: perfil sócio-demográfico da criança, responsável e família, serviços utilizados para reabilitação motora, tipo de demanda, marcação do atendimento, sendo incluídas perguntas abertas sobre dificuldades, pontos positivos e negativos do acesso organizacional, suges-tões para melhorá-lo, cujas respostas foram gravadas e transcritas na íntegra por profissional abalizado; b) prontuários das crianças: características clínicas da PC. Efetuou-se estudo piloto para ajustar instrumento.

As respostas das perguntas pré-codificadas com opção "outras" receberam código específico e as das perguntas abertas (dificuldades identificadas para iniciar e dar continuidade à reabilitação, motivos para interromper a fisioterapia, aspectos positivos na acessibilidade à reabilitação e sugestões para melhorar acessibilidade) foram submetidas à análise de conteúdo de tipo classificatório, com contagem de frequência das categorias. Após leitura flutuante das respostas a uma pergunta determinada, os sentidos/ideias emergentes foram identificados. As ideias que convergiram para um significado comum, com base nos critérios de homogeneidade interna, foram classificados em uma mesma categoria; simultaneamente, buscou-se garantir que as diferenças entre as distintas categorias fossem nítidas e claras, isto é, que houvesse heterogeneidade externa. ${ }^{24}$

Para aumentar a confiabilidade da avaliação recorreu-se: a) à utilização de um referencial teórico consistente apoiando a construção das categorias; e b) ao trabalho de mais de um avaliador, neste caso quatro avaliadores, conciliando divergências de interpretação. Construiu-se banco de dados com programa Epi-info 6.04. As variáveis foram descritas segundo o serviço atual, o primeiro serviço utilizado e o número de serviços utilizados. Dois aspectos mereceram consideração: possibilidade de flu- tuações atribuíveis ao pequeno número de crianças em algumas categorias de respostas e opção em algumas variáveis pela apresentação de praticamente todas as categorias de resposta, evitando o obscurecimento de informações essenciais.

O projeto foi aprovado pelo Comitê de Ética em Pesquisa do Complexo Hospitalar HUOC/ PROCAPE, conforme parecer $\mathrm{n}^{\circ} 113 / 2008$. Os participantes assinaram termo de consentimento livre e esclarecido.

\section{Resultados}

Todos os respondentes pertenciam ao sexo feminino; $94,7 \%$ responsáveis pela criança, sendo $76,3 \%$ a mãe e $18,4 \%$ a avó. Maior percentual tinha entre 20 e 29 anos de idade (42,1\%). A amplitude da distribuição incluiu pessoas entre 20 e 57 anos, com mediana de idade de 31 anos e intervalo entre primeiro e terceiro quartis entre 24 e 42 anos. Da amostra $28,5 \%$ concluíram segundo grau e uma nunca estudou. A amplitude da distribuição abrangeu pessoas de zero a 11 anos de escolaridade, com mediana de sete anos e intervalo entre primeiro e terceiro quartis de cinco a 11 anos.

Constatou-se predomínio de crianças do sexo masculino e forte concentração no grupo etário entre 25 e 59 meses, com 5,3\% tendo menos de um ano de idade. Percentual elevado não dispunha de informação no prontuário sobre tipo de $\mathrm{PC}$, prevalecendo tetraplegia espástica nos casos com registro. Proporção de 71,0\% recebia benefício do Instituto Nacional de Seguridade Social (INSS), 55,5\% dos quais detinham a maior participação no sustento da família (39,5\% do total). Aproximadamente 55,3\% residiam no distrito sanitário de localização do serviço onde foram identificados. Em algum momento da busca por reabilitação motora, 36,8\% não tiveram acesso a serviços. Metade utilizou mais de um serviço, $21 \%$ pelo menos de três. Para $26,3 \%$ houve simultaneidade na utilização de serviços, antes ou durante a coleta de dados (Tabela 1).

Em torno de $73,7 \%$ e $55,3 \%$ das crianças eram menores de um ano quando, respectivamente, receberam diagnóstico de PC e iniciaram reabilitação motora, sendo que $28,2 \%$ tinham mais de seis meses de diagnosticadas ao começar fisioterapia (mediana de 5,3 meses, intervalo entre primeiro e terceiro quartis de 1,5 a 7,5 meses). Aproximadamente $79,0 \%$ chegaram encaminhadas: $75,8 \%$ por médicos pediatras ou neurologistas, $63,3 \%$ de unidades hospitalares/maternidades (predomínio de média e alta densidade tecnológica da rede estadual e instituições públicas de ensino), sendo $83,3 \%$ com referência 
Características sociodemográficas, clínicas e da acessibilidade aos serviços de reabilitação motora da rede complementar da Secretaria de Saúde do Recife das crianças com paralisia cerebral, janeiro a junho de 2009.

\begin{tabular}{|c|c|c|c|c|c|c|c|c|}
\hline \multirow{3}{*}{ Variáveis } & \multicolumn{8}{|c|}{ Serviços } \\
\hline & \multicolumn{2}{|c|}{ CERVAC } & \multicolumn{2}{|c|}{ GURI } & \multicolumn{2}{|c|}{ SER } & \multicolumn{2}{|c|}{ Total } \\
\hline & $\mathrm{n}$ & $\%$ & $\mathrm{n}$ & $\%$ & $\mathrm{n}$ & $\%$ & $\mathrm{n}$ & $\%$ \\
\hline \multicolumn{9}{|l|}{ Sexo } \\
\hline Masculino & 3 & 27,3 & 12 & 50,0 & 2 & 66,7 & 17 & 54,7 \\
\hline Feminino & 8 & 72,7 & 12 & 50,0 & 1 & 33,3 & 21 & 45,3 \\
\hline \multicolumn{9}{|l|}{ Idade (meses) } \\
\hline$\leq 11$ & - & - & 2 & 8,3 & - & - & 2 & 5,3 \\
\hline $12-24$ & 2 & 18,2 & 6 & 25,0 & 2 & 66,7 & 10 & 26,3 \\
\hline $25-59$ & 9 & 81,8 & 16 & 66,7 & 1 & 33,3 & 26 & 68,4 \\
\hline \multicolumn{9}{|l|}{ Tipo de paralisia cerebral } \\
\hline Diplegia espástica & 1 & 9,1 & - & - & - & - & 1 & 2,6 \\
\hline Tetraplegia espástica & - & & 6 & 25,0 & 1 & 33,3 & 7 & 18,4 \\
\hline Mista & - & & 2 & 8,3 & - & - & 2 & 5,3 \\
\hline Sem informação & 10 & 90,9 & 16 & 66,7 & 2 & 66,7 & 28 & 73,7 \\
\hline \multicolumn{9}{|l|}{ Recebe benefício do INSS } \\
\hline Sim & 9 & 81,8 & 17 & 70,8 & 1 & 33,3 & 27 & 71,0 \\
\hline Não & 2 & 18,2 & 7 & 29,2 & 2 & 66,7 & 11 & 29,0 \\
\hline \multicolumn{9}{|c|}{ Maior participação no sustento da família } \\
\hline Sim & 4 & 44,5 & 10 & 58,8 & 1 & 100,0 & 15 & 55,5 \\
\hline Não & 5 & 55,5 & 7 & 41,2 & - & & 12 & 44,5 \\
\hline \multicolumn{9}{|c|}{ Reside no mesmo distrito do serviço atual } \\
\hline Sim & 6 & 54,5 & 15 & 62,5 & - & - & 21 & 55,3 \\
\hline Não & 5 & 45,5 & 9 & 37,5 & 3 & 100,0 & 17 & 44,7 \\
\hline \multicolumn{9}{|c|}{$\begin{array}{l}\text { Acesso à reabilitação motora em todos os } \\
\text { serviços procurados }\end{array}$} \\
\hline Sim & 8 & 72,7 & 13 & 54,2 & 3 & 100,0 & 24 & 63,2 \\
\hline Não & 3 & 27,3 & 11 & 45,8 & - & - & 14 & 36,8 \\
\hline \multicolumn{9}{|c|}{ Número de serviços utilizados } \\
\hline 1 & 3 & 27,2 & 14 & 58,3 & 2 & 66,7 & 19 & 50,0 \\
\hline 2 & 2 & 18,2 & 8 & 33,4 & 1 & 33,3 & 11 & 29,0 \\
\hline 3 & 5 & 45,5 & 2 & 8,3 & - & - & 7 & 18,4 \\
\hline 4 & 1 & 9,1 & - & - & - & - & 1 & 2,6 \\
\hline \multicolumn{9}{|c|}{ Simultaneidade na utilização de serviços } \\
\hline Sim, anteriormente & - & - & 2 & 8,3 & - & - & 2 & 5,3 \\
\hline Sim, atualmente & 4 & 36,4 & 3 & 12,5 & 1 & 33,3 & 8 & 21,0 \\
\hline Não & 7 & 63,6 & 19 & 79,2 & 2 & 66,7 & 28 & 73,7 \\
\hline
\end{tabular}

INSS= Instituto Nacional de Seguridade Social; CERVAC= Centro de Reabilitação e Valorização da Criança; GURI= Grupo Universitário de Reabilitação Infantil; SER= Serviço Especializado de Reabilitação.

documentada. Em 81,6\% dos casos houve deslocamento até serviço para agendar primeira consulta com fisioterapeuta, para $10,5 \%$, a unidade encaminhou e marcou. O tempo de agendamento concentrou-se entre sete e 15 dias, com $86,4 \%$ tendo acesso ao atendimento no máximo em 30 dias. Três referiram como dificuldade de marcação a demora em iniciar o tratamento (Tabela 2).

Em torno de $68,4 \%$ das crianças tiveram alguma dificuldade para entrada à reabilitação, ressaltando a similitude do comportamento a parte a quantidade de serviços utilizados. Falta de vagas, principalmente para as crianças que utilizaram um serviço, seguida da falta de recursos financeiros, foram às dificuldades mais mencionadas. Identificou-se perfil diferenciado quanto às limitações organizacionais, com a carência de fisioterapeutas e os critérios para inscrever a criança representando obstáculos à entrada somente daquelas que utilizaram um serviço (Tabela 3). 
Acessibilidade organizacional no primeiro serviço de fisioterapia motora utilizado por crianças com paralisia cerebral atendidas na rede complementar da Secretaria de Saúde do Recife, janeiro a junho de 2009.

Variáveis

Primeiro serviço utilizado $(\mathrm{N}=38)$

\begin{tabular}{|c|c|c|}
\hline & \multirow[b]{2}{*}{$\mathrm{n}$} & \multirow{2}{*}{$\%$} \\
\hline & & \\
\hline \multicolumn{3}{|l|}{ Idade ao diagnóstico (meses) } \\
\hline$\leq 11$ & 28 & 73,7 \\
\hline $12-24$ & 9 & 23,7 \\
\hline $25-59$ & 1 & 2,6 \\
\hline \multicolumn{3}{|l|}{ Tempo entre diagnóstico e utilização (meses)* } \\
\hline$<1$ & 7 & 21,8 \\
\hline $1-6$ & 16 & 50,0 \\
\hline $7-12$ & 7 & 21,8 \\
\hline $13-24$ & 1 & 3,2 \\
\hline $25-36$ & 1 & 3,2 \\
\hline \multicolumn{3}{|l|}{ Idade ao iniciar tratamento (meses) } \\
\hline$\leq 11$ & 21 & 55,3 \\
\hline $12-24$ & 15 & 39,5 \\
\hline $25-59$ & 2 & 5,2 \\
\hline \multicolumn{3}{|l|}{ Chegou ao serviço } \\
\hline Referido por serviço de saúde & 29 & 76,4 \\
\hline Demanda espontânea & 8 & 21,0 \\
\hline Demanda interna hospitalar & 1 & 2,6 \\
\hline \multicolumn{3}{|l|}{ Serviço que encaminhou } \\
\hline Hospital/Maternidade & 19 & 63,3 \\
\hline Unidade de Saúde da Família & 4 & 13,3 \\
\hline Médico particular & 3 & 10,0 \\
\hline Policlínica & 2 & 6,7 \\
\hline Serviço de reabilitação & 2 & 6,7 \\
\hline \multicolumn{3}{|l|}{ Especialidade do profissional que referiu ${ }^{\dagger}$} \\
\hline Pediatra & 12 & 41,3 \\
\hline Neurologista & 10 & 34,5 \\
\hline Outras especialidades médicas & 6 & 20,7 \\
\hline Equipe Global da AACD & 1 & 3,5 \\
\hline \multicolumn{3}{|l|}{ Referência foi documentada } \\
\hline $\operatorname{Sim}$ & 25 & 83,3 \\
\hline Não & 5 & 16,7 \\
\hline \multicolumn{3}{|l|}{ Marcação da primeira consulta } \\
\hline Responsável/rede de apoio foi agendar & 31 & 81,6 \\
\hline Unidade marcou & 4 & 10,5 \\
\hline Responsável/rede de apoio marcou por telefone & 3 & 7,9 \\
\hline \multicolumn{3}{|l|}{ Tempo de agendamento (dias) $\neq$} \\
\hline $1-6$ & 8 & 21,6 \\
\hline $7-14$ & 15 & 40,5 \\
\hline $15-30$ & 9 & 24,3 \\
\hline $31-120$ & 5 & 13,6 \\
\hline \multicolumn{3}{|l|}{ Dificuldade de marcação } \\
\hline Sim, intervalo para iniciar tratamento & 3 & 7,9 \\
\hline Não & 35 & 92,1 \\
\hline
\end{tabular}

AACD=Associação de Assistência a Criança Deficiente;

* Seis crianças iniciaram tratamento antes do diagnóstico de paralisia cerebral: duas referidas com

diagnóstico inicial de mielomeningocele e quatro por demanda espontânea;

† Uma criança sem informação sobre especialidade do profissional que referiu;

‡ Uma criança sem informação sobre o tempo de agendamento. 
Características sociodemográficas e obstáculos à acessibilidade organizacional das crianças com paralisia cerebral atendidas na rede complementar da Secretaria de Saúde do Recife, segundo número de serviços utilizados, janeiro a junho de 2009.

\begin{tabular}{|c|c|c|c|c|}
\hline \multirow{3}{*}{ Variáveis } & \multicolumn{4}{|c|}{ Número de serviços utilizados } \\
\hline & \multicolumn{2}{|c|}{ Um } & \multicolumn{2}{|c|}{ Dois a quatro } \\
\hline & $\mathrm{n}$ & $\%$ & $\mathrm{n}$ & $\%$ \\
\hline \multicolumn{5}{|l|}{ Idade (meses) } \\
\hline $0-24$ & 5 & 26,3 & 7 & 36,9 \\
\hline $25-59$ & 14 & 73,7 & 12 & 63,1 \\
\hline \multicolumn{5}{|l|}{ Recebe benefício do INSS } \\
\hline Sim & 14 & 73,7 & 13 & 68,4 \\
\hline Não & 5 & 26,3 & 6 & 31,6 \\
\hline \multicolumn{5}{|l|}{ Dificuldades na entrada à reabilitação motora } \\
\hline Sim & 15 & 78,9 & 11 & 57,9 \\
\hline Não & 4 & 21,9 & 8 & 42,1 \\
\hline \multicolumn{5}{|l|}{ Principais dificuldades identificadas* } \\
\hline Falta de vagas/excesso de demanda & 9 & 60,0 & 4 & 36,4 \\
\hline Recursos financeiros para transporte & 4 & 26,7 & 3 & 27,3 \\
\hline Falta de diagnóstico & 1 & 6,7 & 3 & 27,3 \\
\hline Disponibilidade de fisioterapeutas & 4 & 26,7 & - & - \\
\hline Critérios para inscrição da criança & 3 & 20,0 & - & - \\
\hline Disponibilidade de serviços & 1 & 6,7 & 1 & 9,1 \\
\hline Falta de informação sobre serviços & 1 & 6,7 & 1 & 9,1 \\
\hline Critérios para desligamento da criança & - & - & 1 & 9,1 \\
\hline \multicolumn{5}{|l|}{ Dificuldade para continuar tratamento } \\
\hline Sim & 2 & 10,5 & 15 & 78,9 \\
\hline Não & 17 & 89,5 & 4 & 21,1 \\
\hline \multicolumn{5}{|l|}{ Principais dificuldades encontradas* } \\
\hline Distância residência/serviço & 1 & 50,0 & 7 & 46,7 \\
\hline Transporte público & 1 & 50,0 & 5 & 33,3 \\
\hline Peso da criança & 1 & 50,0 & 4 & 26,7 \\
\hline Horário da fisioterapia & - & - & 4 & 26,7 \\
\hline Financiamento do transporte & 1 & 50,0 & 2 & 13,3 \\
\hline Obtenção de metas terapêuticas & - & - & 2 & 13,3 \\
\hline Tempo de espera por atendimento & - & - & 2 & 13,3 \\
\hline Qualidade técnica insatisfatória & - & - & 2 & 13,3 \\
\hline Normas do serviço para desligamento & - & - & 2 & 13,3 \\
\hline Mudança de fisioterapeuta & - & - & 1 & 6,7 \\
\hline
\end{tabular}

*Distintas razões para as dificuldades na entrada à reabilitação e continuidade do tratamento, motivo pelo qual a soma é superior a $100 \%$; INSS= Instituto Nacional de Seguridade Social.

Metade das crianças interrompeu o tratamento fisioterápico, sendo a principal razão de descontinuidade da fisioterapia o adoecimento da criança, independentemente da quantidade de serviços. Todavia, enquanto para as crianças que utilizaram dois e mais serviços os motivos de interrupção contemplaram aspectos relacionados à organização dos serviços e recursos financeiros, para aquelas que utilizaram um serviço foram destacados elementos atinentes à mãe e à rede informal de apoio (Tabela 4).

Ao redor de $92,0 \%$ identificaram aspectos positivos no acesso. A resposta da criança ao tratamento fisioterapêutico foi o elemento mais valorizado para aquelas que utilizaram um serviço, embora tenha sido importante para o outro grupo. Para crianças que utilizaram de dois a quatro serviços, os aspectos mais citados foram facilidade de acesso à 
Aspectos positivos/ negativos e sugestões dos responsáveis para melhorar acessibilidade à reabilitação motora das crianças com paralisia cerebral atendidas na rede complementar da Secretaria de Saúde do Recife, segundo número de serviços utilizados, janeiro a junho de 2009.

\begin{tabular}{|c|c|c|c|c|}
\hline \multirow{3}{*}{ Variáveis } & \multicolumn{4}{|c|}{ Número de serviços utilizados } \\
\hline & \multicolumn{2}{|c|}{ Um } & \multicolumn{2}{|c|}{ Dois a quatro } \\
\hline & $\mathrm{n}$ & $\%$ & $\mathrm{n}$ & $\%$ \\
\hline \multicolumn{5}{|l|}{ Houve interrupção da fisioterapia } \\
\hline Sim & 11 & 57,9 & 8 & 42,1 \\
\hline Não & 8 & 42,1 & 11 & 57,9 \\
\hline \multicolumn{5}{|l|}{ Motivos para interromper fisioterapia* } \\
\hline Doença da criança & 6 & 54,5 & 6 & 75,0 \\
\hline Dificuldades financeiras & - & - & 4 & 50,0 \\
\hline Falta de apoio da rede & 3 & 27,3 & - & - \\
\hline Serviço em recesso & - & - & 3 & 37,5 \\
\hline Desligamento do serviço & 1 & 9,1 & 2 & 25,0 \\
\hline Doença materna & 2 & 18,2 & - & - \\
\hline Mãe não levava criança & 2 & 18,2 & - & - \\
\hline Outros & - & - & 3 & 37,5 \\
\hline \multicolumn{5}{|c|}{ Aspectos positivos no acesso à reabilitação motora } \\
\hline Sim & 16 & 84,2 & 19 & 100,0 \\
\hline Não & 3 & 15,8 & - & \\
\hline \multicolumn{5}{|l|}{ Principais aspectos positivos na acessibilidade } \\
\hline Resposta da criança ao tratamento & 9 & 56,2 & 6 & 31,6 \\
\hline Facilidade de acesso & 1 & 6,2 & 7 & 36,8 \\
\hline Qualidade técnica da atenção & 3 & 18,7 & 4 & 21,0 \\
\hline Qualidade humana da atenção & 2 & 12,5 & 5 & 26,3 \\
\hline Acesso à consulta médica & - & - & 4 & 21,0 \\
\hline Acesso às órteses & 1 & 6,2 & 1 & 5,3 \\
\hline Orientações sobre cuidados da criança & 1 & 6,2 & - & - \\
\hline \multicolumn{5}{|l|}{ Sugestões para melhorar o acesso a serviços } \\
\hline Sim & 12 & 63,1 & 16 & 84,2 \\
\hline Não & 7 & 46,9 & 3 & 15,8 \\
\hline \multicolumn{5}{|l|}{ Principais sugestões apresentadas§ } \\
\hline Aumentar número de profissionais & 5 & 41,7 & 6 & 37,5 \\
\hline Aumentar número de sessões & 4 & 33,3 & 3 & 18,7 \\
\hline Aumentar tempo de cada sessão & 4 & 33,3 & 3 & 18,7 \\
\hline Ampliar número de vagas & - & - & 5 & 31,2 \\
\hline Acesso independente do quadro clínico & - & - & 3 & 18,7 \\
\hline Oferta da atividade em postos de saúde & 3 & 25,0 & 1 & 6,2 \\
\hline Disponibilidade de transporte pelo serviço & - & - & 4 & 25,0 \\
\hline Ofertar outras atividades & 2 & 16,7 & 1 & 6,2 \\
\hline
\end{tabular}

* Vários motivos foram apresentados para interrupção da fisioterapia, razão pela qual a soma é superior a $100 \%$.

\# Distintas razões foram apresentadas para os aspectos positivos, motivo pelo qual a soma é superior a $100 \%$.

$\S$ Várias sugestões foram apresentadas, razão pela qual a soma é superior a $100 \%$.

fisioterapia, além da qualidade humana e técnica da atenção. Verifica-se que $73,7 \%$ deram sugestões para melhorar acesso, sendo o aumento do número de fisioterapeutas a mais frequente, seguida, para crianças que utilizaram um serviço, do aumento do número e tempo das sessões de fisioterapia motora. Para o outro grupo, da ampliação do número de vagas, disponibilidade de transporte pelo serviço e aumento do número e tempo das sessões (Tabela 4). 


\section{Discussão}

Observa-se a justaposição de características que mostraram as precárias condições de vida das crianças estudadas, tais como a própria criança detendo a maior participação no sustento da família e as pessoas responsáveis, todas do sexo feminino, com predomínio daquelas que não concluíram o primeiro grau de escolaridade. Nesse contexto, chama a atenção o fato de que uma maior proporção de crianças foi diagnosticada e iniciou tratamento reabilitador antes de completar um ano de idade. Comportamento que diverge da literatura, na qual é reconhecida a associação entre baixo nível socioeconômico, baixa escolaridade e menor percepção da necessidade de utilizar os serviços de saúde entre cuidadores de crianças com deficiência. 25 Este desacordo, certamente, pode resultar do pequeno número de participantes na presente investigação.

Diferentemente dos resultados de pesquisas que constatam que, com frequência, não eram os médicos que estavam referindo as crianças com deficiência para os serviços de reabilitação, neste estudo, praticamente todas as crianças encaminhadas para fisioterapia obedeciam à conduta prescrita por médicos, sobretudo pediatras e neurologistas. Aspecto indicativo, apesar da omissão de registro nos prontuários infantis, da possibilidade de uma maior precisão no diagnóstico e de uma percepção mais acurada da utilização dos recursos terapêuticos que aumentou a probabilidade do inicio precoce da reabilitação motora. 15,18

Vários autores afirmam que crianças com deficiências graves tendem a ser referidas mais precocemente.15,18 No estudo atual, alguns elementos apontam a possibilidade de que prevaleçam formas mais graves de PC: excluída uma, todas as crianças com informação sobre diagnóstico apresentavam tetraplegia; o adoecimento decorrente da labilidade clínica foi importante motivo para interrupção temporária da fisioterapia e as críticas negativas dos critérios institucionais que dificultavam a inclusão e continuidade do tratamento. Encontra-se bem estabelecido que as formas mais severas de deficiência originadas deste agravo concentram-se nas famílias de baixo nível socioeconômico. 23

Em consonância com outras pesquisas, ${ }^{20,26}$ os critérios adotados pelos serviços de reabilitação levaram as crianças a um acesso seletivo à fisioterapia motora. Conforme denotaram as respostas das responsáveis das crianças que utilizaram dois e mais serviços acerca dos motivos de interrupção do tratamento reabilitador (desligamento do serviço), das dificuldades para continuar fisioterapia (obtenção de metas terapêuticas) e das sugestões para melhorar acesso à reabilitação motora (acesso independente do quadro clínico), bem como das responsáveis daquelas que utilizaram um serviço, quanto às dificuldades para acesso à reabilitação (critérios para inscrição da criança).

As limitações organizacionais decorrentes dos critérios para atender as necessidades provocadas pela PC reiteram o descrito na literatura internacional: apesar da tendência ao encaminhamento mais precoce, as crianças com deficiências graves podem ter o acesso negado quando inexiste expectativa de melhora significativa em curto prazo. ${ }^{15,18}$ Essas restrições são sugestivas de que a demanda é maior do que a oferta de fisioterapia motora, ${ }^{19}$ provocando, necessariamente, à discussão sobre equidade e acesso.14,27 Por outro lado, cabe lembrar que a dificuldade de avaliação do progresso entre as crianças com incapacidade física grave torna-se maior pela falta de instrumentos sensíveis para detectar pequenas mudanças na habilidade motora. 28

Proporção elevada das crianças teve um período de espera de 30 dias para realizar a primeira consulta com fisioterapeuta, configurando-se como um intervalo de tempo curto em relação aos dois estudos efetuados em Montreal.17,18 Nestes, as crianças portadoras de deficiência esperaram, em média, quatro e sete meses entre o agendamento e o atendimento de fisioterapia motora. Todavia, na cidade do Recife, 28,2\% não haviam iniciado fisioterapia motora seis meses após o diagnóstico de PC. Embora a percepção das necessidades especiais pelos responsáveis da criança com deficiência possa retardar o acesso à reabilitação 8,15 -18 convém atentar-se que nem sempre o primeiro serviço utilizado correspondeu ao primeiro procurado, evidenciando a demanda reprimida (excesso de demanda, falta de vagas) mais mencionada quando a criança utilizou um único serviço.

O relatório da $8^{\mathrm{a}}$ Conferência Municipal de Saúde, 29 realizada em 2007, demonstra que a garantia da atenção às pessoas com necessidades de reabilitação é uma das propostas da gestão. Entretanto, embora esteja em curso à implantação do sistema de referência e contrarreferência ambulatorial da rede de serviços municipais, o mecanismo de agendamento estabelecido pela central de regulação do município ainda não abrange a maior parte das unidades de saúde que diagnosticam os casos de PC. Por isso, em geral, coube às famílias o deslocamento até o serviço para marcar a primeira consulta com fisioterapeuta na rede de serviços complementares do SUS. Isto, em um contexto onde a falta de recursos financeiros para transporte representou 
motivo de interrupção temporária da fisioterapia, dificuldade para iniciar e dar continuidade à reabilitação motora.

Não se pode tratar da acessibilidade organizacional sem levar em conta o grau de ajuste entre as necessidades dos pacientes e os serviços e recursos utilizados. 10,12,13 Aspectos tradutores do reconhecimento da adequação do tratamento, o progresso alcançado pela criança e a qualidade técnica da fisioterapia foram pontos positivos de enorme relevância na avaliação das responsáveis. Contudo, as sugestões para melhorar acesso à reabilitação motora, embora com menor frequência, manifestaram a insatisfação com a qualidade técnica, solicitando o aumento do número de sessões e o tempo de cada sessão. Num contexto de demanda reprimida e necessidade de utilização dos serviços de reabilitação por um longo tempo, torna-se relevante a melhoria do acompanhamento, inclusive, oferecendo sessões mais longas e mais freqüentes. 8,21

As percepções e juízos das responsáveis por estas crianças sobre a qualidade da assistência condicionam a busca (ou não) de outras fontes de atenção. A superposição na utilização de serviços é também um forte indicativo da insatisfação com a assistência prestada na reabilitação motora. Nesse sentido, a simultaneidade do uso pode ser considerada como uma estratégia para solucionar as necessidades não satisfeitas. Ortega et al.30 mencionam a possibilidade de que a insatisfação com a qualidade da atenção origine padrões de utilização ineficientes, com múltiplos prestadores e falta de coordenação dos cuidados.

No processo de organização do SUS, a descentralização mediante regionalização e hierarquização da assistência, é condição necessária para melhorar a acessibilidade à atenção, sobretudo a acessibilidade geográfica, à medida que busca garantir uma

\section{Referências}

1. Bernardes LCG, Maior IMML, Spezia CH. Pessoas com deficiência e políticas de saúde no Brasil: reflexões bioéticas. Ciênc Saúde Coletiva. 2009; 14: 31-8.

2. IBGE (Instituto Brasileiro de Geografia e Estatística) Censo Demográfico 2000: características da população e dos domicílios: resultados do universo. Brasil; 2000.

3. Mohammed MSJ. Cerebral palsy: comprehensive review and update. Ann Saudi Med. 2006; 26: 123-32.

4. Vincer MJ, Allen AC, Joseph KS, Stinson DA, Scott H, Wood E. Increasing prevalence of cerebral palsy among very preterm infants: a population-based study. Pediatrics. 2006; 118: 1621-6. distribuição espacial que viabilize a utilização dos recursos pelos usuários. ${ }^{22}$ Neste sentido, a proposta da $8^{\text {a }}$ conferência para, nos seis distritos sanitários, implantar núcleos de referência de reabilitação (fisioterapia, terapia ocupacional, fonoaudiologia), articulados a partir do Núcleo de Apoio à Saúde da Família (NASF) e expandir os serviços de referência de acordo com a demanda, qualificando os já existentes. ${ }^{29}$ A distância entre a residência e o serviço foi, isoladamente, à dificuldade mais citada para continuidade do tratamento, despontando a reivindicação das responsáveis das crianças por uma maior proximidade com serviços.

Para assegurar a acessibilidade funcional aos serviços de reabilitação motora torna-se imprescindível uma maior valorização dos processos políticos e organizacionais voltados para estruturação do sistema de referência e contrarreferência. Logo, é preciso conhecer a gama de serviços disponíveis e sua complementaridade, os vínculos existentes entre os serviços e a pertinência desses serviços em relação às necessidades das crianças portadoras de deficiência física, dentre as quais se encontram aquelas com PC, criando espaços de negociação para explorar novas soluções. 30

É conveniente lembrar que, neste estudo, apesar das restrições decorrentes do fato do método quantitativo minimizar a experiência subjetiva, buscou-se reduzir uma limitação importante desse tipo de abordagem, na medida em que foram consideradas as percepções dos responsáveis pela criança para julgar a experiência relacionada com a acessibilidade à fisioterapia. Portanto, para identificar, num determinado contexto, tanto os aspectos que estão associados ao sentir-se com os valores e expectativas correspondidos, quanto às mudanças que precisam ser efetuadas para assegurar a acessibilidade aos serviços.

5. Mancini MC, Fiúza PM, Rebelo JM, Magalhães LC, Coelho ZAC, Paixão ML, Gontijo APB, Fonseca ST. Comparação do desenvolvimento de atividades funcionais em crianças com desenvolvimento normal e crianças com paralisia cerebral. Arq Neuro-Psiquiatr. 2002; 60: 446-52.

6. Koman LA, Smith BP, Shilt JS. Cerebral Palsy. Lancet. 2004; 363: 1619-31.

7. Rotta NT. Paralisia cerebral, novas perspectivas terapêuticas. J Pediatr (St. Louis). 2002; 78 (Supl. 1): 48-54.

8. Elrod CS, DeJong G. Determinants of utilization of physical rehabilitation services for persons with chronic and disabling conditions: an exploratory study. Arch Phys Med Rehabil. 2008; 89: 114-20. 
9. Brasil. Ministério da Saúde. Secretaria de Assistência à Saúde. Coordenação de Atenção a Grupos Especiais. Programa Nacional de Saúde da Pessoa Portadora de Deficiência. [Acesso 19 jul 2008]. Disponível em: http://portal.saude.gov.br/portal/arquivos/pdf/manual2.pdf

10. Travassos C, Martins M. Uma revisão sobre os conceitos de acesso e utilização de serviços de saúde. Cad Saúde Pública. 2004; 20: 190-8

11. Donabedian A. The seven pillars of quality. Arch Pathol Lab Med. 1990; 114: 1115-8.

12. Ramos DD, Lima MAS. Acesso e acolhimento aos usuários em uma unidade de saúde de Porto Alegre, Rio Grande do Sul, Brasil. Cad Saúde Pública. 2003; 19: 27-34.

13. Fekete MC. Estudo da acessibilidade na avaliação dos serviços de saúde. In: Santana JP, org. Desenvolvimento Gerencial de Unidades Básicas do Sistema Único de Saúde (SUS). Brasília: OPS; 1997.

14. Franco SC, Campos GWS. Acesso a ambulatório pediátrico de um hospital universitário. Rev Saúde Pública. 1998; 32: 352-60.

15. Majnemer A, Shevell ML, Rosenbaum P, Abrahamowicz M. Early rehabilitation service utilization patterns in young children with developmental delays. Child Care Health Dev. 2002; 28: 29-37.

16. Feldman DE, Champagne F, Bitensky NK, Meshefedjian G Rehabilitation services for physically disabled children in Montreal. Academy for Health Services Research and Health Policy Meet. 2001; 18: 70

17. Feldman E, Champagne F, Korner-Bitensky N, Meshefedjian G. Waiting time for rehabilitation services for children with physical disabilities. Child Care Health Dev. 2002; 28: 351-8

18. Grilli L, Feldman DE, Swaine B, Gosselin J, Champagne F, Pineault R. Wait times for paediatric rehabilitation. Healthcare Policy 2007; 2: e171-87.

19. Meio MDBB, Magluta C, Mello RR, Moreira MEL. Análise situacional do atendimento ambulatorial prestado a recémnascidos egressos das unidades de terapia intensiva neonatais no Estado do Rio de Janeiro. Ciênc Saúde Coletiva. 2005; 10: 299-307.

Recebido em 29 de junho de 2011

Versão final apresentada em 3 de outubro de 2011

Aprovado em 31 de outubro de 2011
20. Bradley SF, Elena A, Kristofer H. Disability and satisfaction with access to health care. J Epidemiol Community Health. 2000; 54: 770-1.

21. Chan HS, Lau PH, Fong KH, Poon D, Lam CC. Neuroimpairment, activity limitation, and participation restriction among children with cerebral palsy in Hong Kong. Hong Kong Medical J. 2005; 11: 342-50.

22. Beckung E, Hagberg G. Neuroimpairments, activity limitations and participation restrictions in children with cerebral palsy. Dev Med Child Neurol. 2002; 44: 309-16.

23. Odding E, Roebroeck ME, Stam HJ. The epidemiology of cerebral palsy: incidence, impairments and risk factors. Disabil Rehabil. 2006; 28: 183-91.

24. Bardin L. Análise de respostas a questões abertas: a simbólica do automóvel. In: Análise de Conteúdo. Lisboa (Portugal): Edições 70; 1977. p. 59-63.

25. Porterfield SL, McBride TD. The effect of poverty and caregiver education on perceived need and access to health services among children with special health care needs. Am J Public Health. 2007; 97: 323-9.

26. França ISX, Pagliuca LMF, Baptista RS. Política de inclusão do portador de deficiência: possibilidades e limites. Acta Paul Enferm. 2008; 21: 112-6.

27. Adam O, Elias M. Equity of access to health care: outlining the foundations for action. J Epidemiol Community Health. 2004; 58: 655-8.

28. Siebes RC, Wijnroks L, Vermeer A. Qualitative analysis of therapeutic motor intervention programmes for children with cerebral palsy: an update. Dev Med Child Neurol. 2002; 44: 593-603.

29. Pernambuco. Prefeitura do Recife. Relatório da $8^{\mathrm{a}}$ Conferência Municipal de Saúde-2007: pacto pela saúde com controle social - fortalecendo o SUS e promovendo a qualidade de vida. Recife; 2007.

30. Ortega JL, Infante C, Palacios ET. A duplicação de serviços como expressão de insatisfação dos pacientes. In: Bosi MLM, Mercado FJ, orgs. Pesquisa qualitativa de serviços de saúde. Petrópolis (RJ): Vozes; 2004. p. 337-62. 